\title{
Calibration of a Stereo Radiation Detection Camera Using Planar Homography
}

\author{
Seung-Hae Baek, Pathum Rathnayaka, and Soon-Yong Park \\ School of Computer Science and Engineering, Kyungpook National University, Daegu 702-701, Republic of Korea \\ Correspondence should be addressed to Soon-Yong Park; sypark@knu.ac.kr
}

Received 20 February 2015; Revised 3 May 2015; Accepted 4 May 2015

Academic Editor: Hocine Cherifi

Copyright ( 12016 Seung-Hae Baek et al. This is an open access article distributed under the Creative Commons Attribution License, which permits unrestricted use, distribution, and reproduction in any medium, provided the original work is properly cited.

\begin{abstract}
This paper proposes a calibration technique of a stereo gamma detection camera. Calibration of the internal and external parameters of a stereo vision camera is a well-known research problem in the computer vision society. However, few or no stereo calibration has been investigated in the radiation measurement research. Since no visual information can be obtained from a stereo radiation camera, it is impossible to use a general stereo calibration algorithm directly. In this paper, we develop a hybrid-type stereo system which is equipped with both radiation and vision cameras. To calibrate the stereo radiation cameras, stereo images of a calibration pattern captured from the vision cameras are transformed in the view of the radiation cameras. The homography transformation is calibrated based on the geometric relationship between visual and radiation camera coordinates. The accuracy of the stereo parameters of the radiation camera is analyzed by distance measurements to both visual light and gamma sources. The experimental results show that the measurement error is about $3 \%$.
\end{abstract}

\section{Introduction}

Detection of radioactive sources is one of the important topics in many research areas, particularly in nuclear instrumentation, nuclear physics, nuclear medicine, and many nondestructive tests [1]. Radiation detectors, also known as particle detectors, are used to detect, track, and identify the presence of radioactive sources within a given area or environment. As a generic definition, radiation is an energy that comes from a source and travels through some material or space. They can be found in many different formats such as alpha, beta, gamma, and X-ray. The detection of sources that emit radiation plays a very significant role in many areas of science and industry. Mainly, gamma radiation has managed to attract the interest of most of the scientists since the advance of the Anger camera [2].

Not only the detection of radioactive sources is crucial, but also the accurate estimation of their three-dimensional (3D) information is important. If the 3D position information of radioactive (such as gamma) sources is available, their activity (strength) can be estimated more precisely. However, detection of gamma sources which are widely distributed in the 3D environment is not an easy task to accomplish as it may seem. Determining or estimating the 3D coordinates of such widely spread gamma sources using a single camera is almost impossible. Therefore, in this paper, we use a stereo gamma detection device instead of using mono devices to estimate their coordinates precisely.

When detecting and extracting metric information from two-dimensional (2D) radiation images using a stereo gamma camera (SGC), we must consider the calibration of the camera. Proper calibration methods have to be implemented in order to obtain more accurate $3 \mathrm{D}$ coordinate measurement. Since accurate calibration of vision cameras is of interest in computer vision, many related works have been done throughout the last few decades [3-7].

Although much work related to the calibration of stereo and mono vision camera has been introduced, a proper calibration method of an SGC has not been introduced yet. Considering it as a fact, we propose a new simplified method to calibrate such SGC devices using planar homography relationships. The experiment system consists of a $1 \mathrm{D}$ gamma sensor and a 2D vision sensor, which are mounted on a pantilt motion device. Because the gamma sensor is a $1 \mathrm{D}$ point 


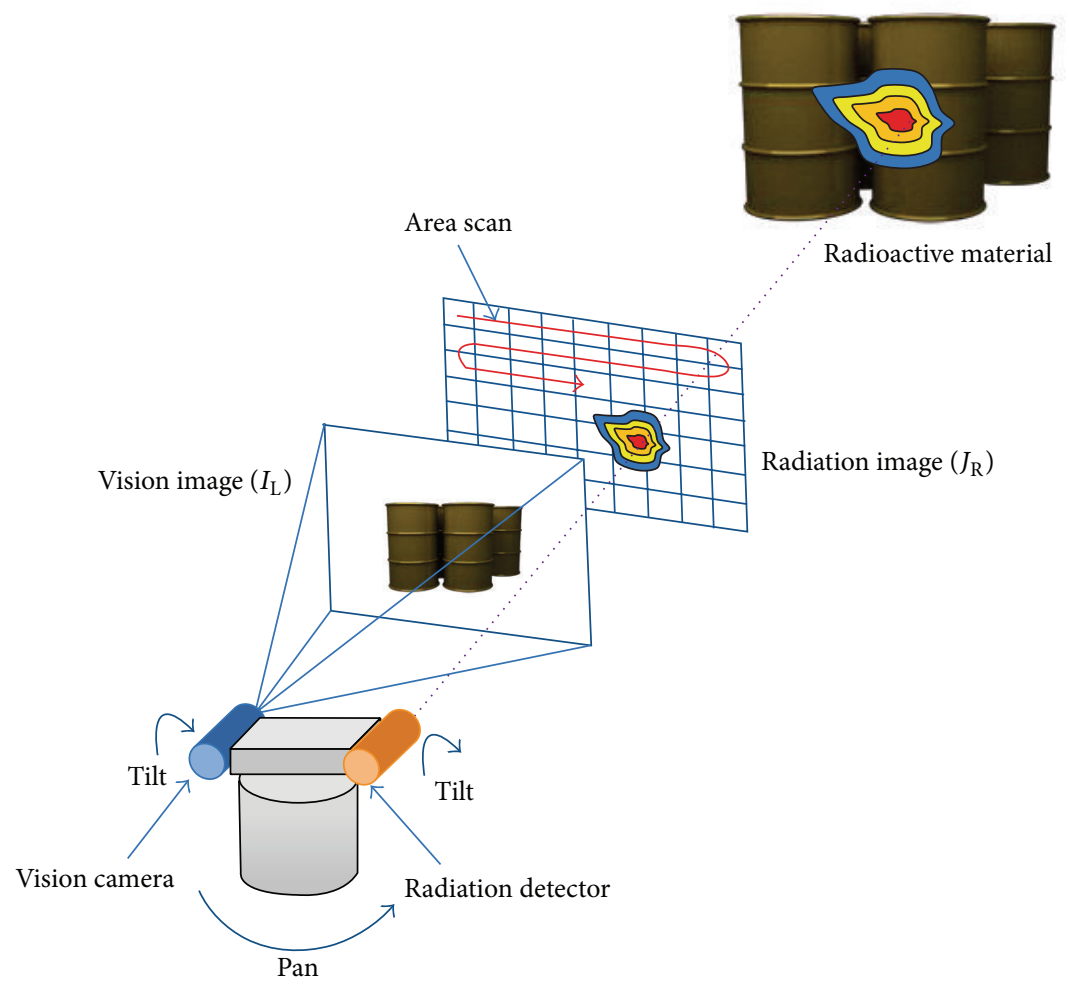

FIGURE 1: Obtaining a gamma image by using a gamma detector and a pan-tile module.

sensor, the pan-tilt device scans the sensor in horizontal and vertical directions to acquired $2 \mathrm{D}$ gamma images. In addition, the pan-tilt device also rotates both the gamma sensor and vision sensor in a symmetric position so that each sensor can obtain stereo images with the same baseline. Therefore the gamma sensor combined with the pan-tilt device works as a $2 \mathrm{D}$ radiation camera and the vision camera is used to acquire the corresponding $2 \mathrm{D}$ visual information.

To measure 3D information from the stereo gamma images, the stereo gamma camera must be calibrated. However, no visual information can be obtained from the camera, and we transform the visual images obtained from the vision camera to the view of the gamma camera. In this way, we can obtain virtual calibration images of the gamma camera to calibrate the stereo geometry of the gamma camera. Once the devices are fully calibrated, we can measure the parallax in the stereo gamma images and distance to the radioactive sources.

The paper is constructed as follows. Section 2 introduces the panning and tilting method implemented to generate radiation images. Section 3 describes the method used to calculate the planar homography relationships between gamma camera images and vision camera images. The proposed calibration method is described in Section 4. Section 5 presents the experiments we have performed to measure 3D distance to both visual light and gamma sources. In this section, we evaluate the accuracy of the proposed calibration method by comparing the acquired results with real data. Section 6 consists with the conclusion.

\section{Obtaining Stereo Gamma Images Using a Gamma Detector}

The approach of obtaining gamma images is based on scanning a $1 \mathrm{D}$ gamma detector using a pan-tilt motion module. Figure 1 shows a simplified diagram of this concept. A 1D gamma detector is mounted on a pan-tilt module which is connected to a general purpose PC. The rotation of the pantilt module in panning and tilting directions is controlled by a terminal program of the PC. According to the resolution of a gamma image, the sensing direction of the pan-tilt module is determined. At every sensing direction, the detector obtains the corresponding pixel's grey value of the image. By obtaining all pixel values, a 2D gamma image is then generated. Therefore, we can consider that there is a virtual 2D gamma camera which can capture a gamma image.

As a gamma detector, a SPMT (small photo multiplier tube) is used with a $\mathrm{Nal}(\mathrm{Tl})$ type scintillator. The detection sensitivity of the SPMT is the maximum at $420 \mathrm{~nm}$. The output type of the detector is the pulse count of photons, which are generated by the SPMT. For more information, see reference [8]. The pulse counts from the SPMT are recorded and mapped to the grey value of a pixel in the gamma image.

A $2 \mathrm{D}$ vision camera is also mounted on the opposite side of the gamma detector. The vision camera only captures two ways of visual information, the left and the right images, of the scanning area. The viewing angle of the vision camera is determined by the lens of the camera and it is usually different than the scanning area of the detector. However, it can be 


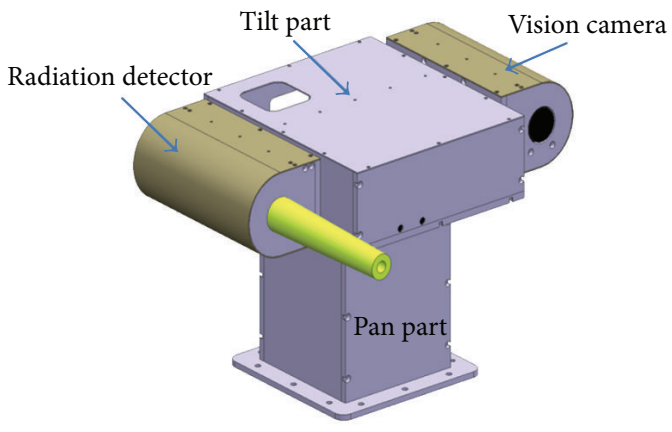

(a)

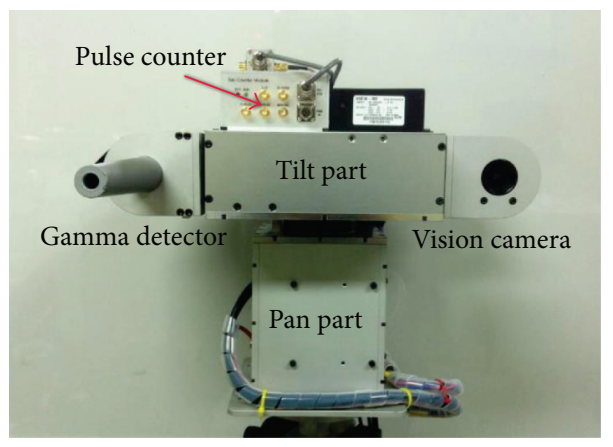

(b)

FIGURE 2: (a) CAD model and (b) real view of the pan-tilt module and two enclosures for mounting gamma and vision cameras.

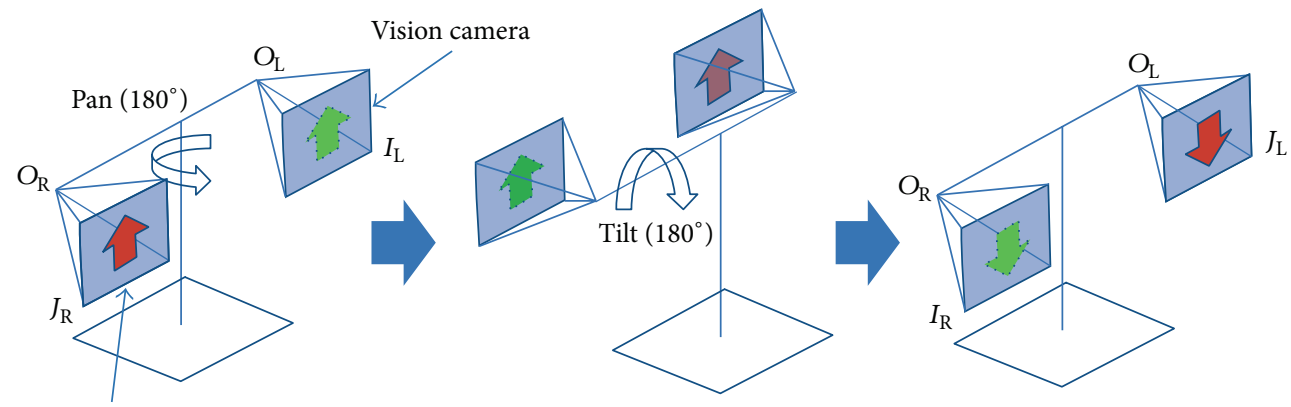

Gamma camera

FIgURE 3: Panning and tilting of sensing devices for obtaining stereo gamma and vision images.

transformed to be matched with the scanning area of the detector by using the calibration parameters of the camera.

Figure 2 shows a CAD model and a real view of the pan-tile module equipped with two enclosures for mounting gamma and vision camera. The pan part of the module rotates the tilt part and two sensing devices more than 180 degrees. In addition, the tilt part rotates the gamma detector and the vision camera more than 180 degrees also. Figure 3 shows diagrams to explain the way of capturing stereo images of two sensing devices. In the beginning, suppose the gamma camera is on the right side of the pan-tile module and the vision camera is on the left side. Therefore, we can obtain the right gamma image $\left(J_{\mathrm{R}}\right)$ from the virtual gamma camera and the left vision image $\left(I_{\mathrm{L}}\right)$ from the vision camera. Next, let us rotate the pan and tilt parts 180 degrees, respectively. Then the origin of the sensor coordinate systems is exactly exchanged, while the image planes of the cameras are rotated 180 degrees with respect to the image center. The image rotation can be easily rotated to the right directions; finally, we can obtain the left gamma image $\left(J_{L}\right)$ and the right vision image $\left(I_{R}\right)$ in the final position.

In order to rotate the pan-tilt module accurately, we control the module using very accurate DC motors and a motion controller. The motors are equipped with encoders with the resolution of 2048 pulses per revolution. In addition the rotation of the pan and tilt module is reduced by $100: 1$ gear ratio. Therefore, the rotation accuracy is very high and we assume the error is less than 0.01 degrees. Table 1 shows the specification of some categories of the pan-tile module.
TABLE 1: Specifications of the pan-tilt module.

\begin{tabular}{lc}
\hline Category & Specification \\
\hline Baseline $\left(\left|O_{\mathrm{L}}-\mathrm{O}_{\mathrm{R}}\right|\right)$ & $280 \mathrm{~mm}$ \\
Pan/tilt encoder resolution & $2048 \mathrm{pulse} / \mathrm{rev}$ \\
Pan/tilt gear ratio & $100: 1$ \\
Rotation accuracy & $<0.01^{\circ}$ \\
Mechanical backlash & $<0.1^{\circ}$ \\
Dimension & $360 \times 270 \times 200 \mathrm{~mm}^{3}(W \times H \times D)$
\end{tabular}

\section{Homography Relationship between Gamma and Vision Cameras}

The first step of the proposed method is calculating the homography relationship between two different image planes of the gamma and vision cameras. Currently two types of gamma detectors are used: pin-hole and coded aperture types [9]. As described in the previous section, our gamma sensor is a pin-hole detector which consists of a cylindrical collimator and an SPMT. Assuming that the gamma and vision cameras follow the pinhole camera model, the image formation between the gamma and vision cameras is related by a homography. In the previous section, we show that the coordinate origins of the gamma and vision cameras are the same because of the symmetric motion of the pantilt module. There are two homography relations between gamma and vision images: one is between left gamma and 


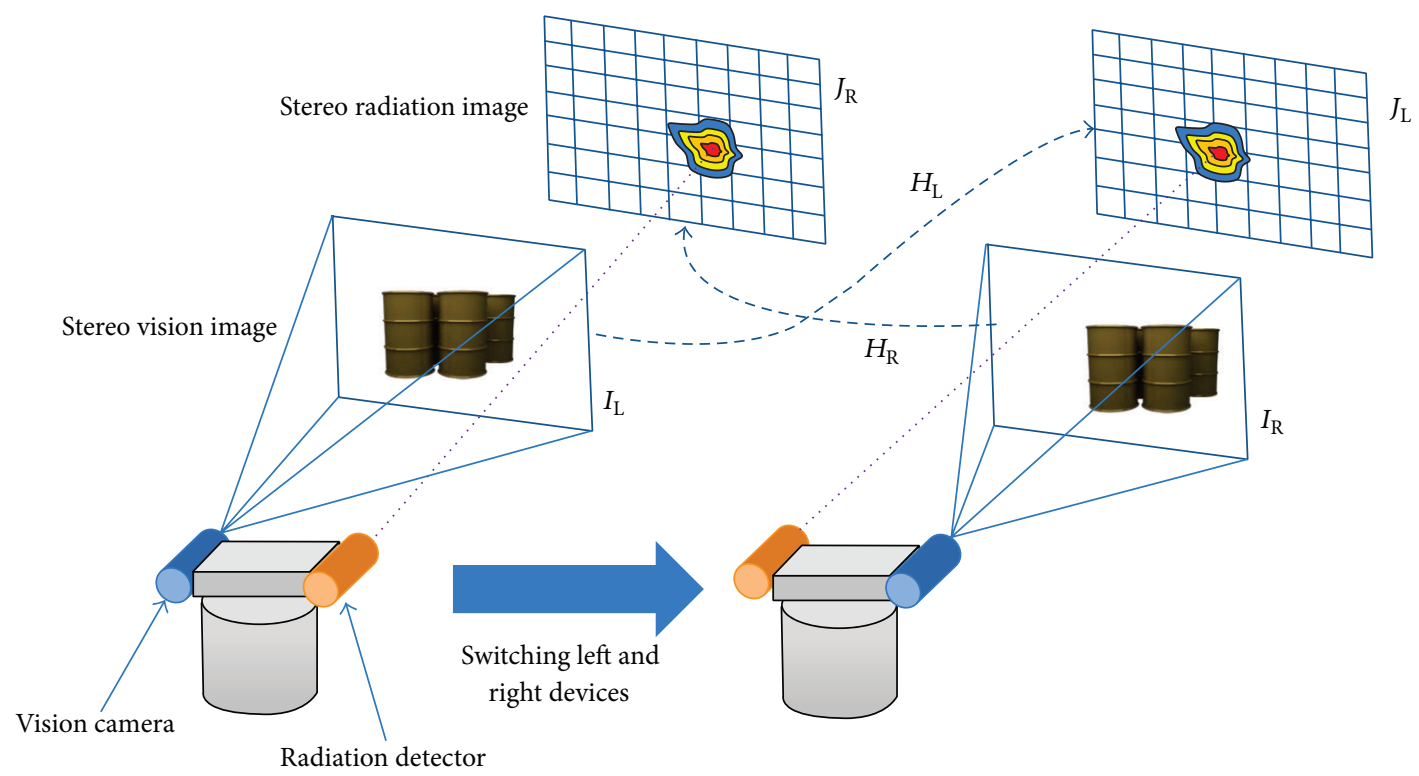

FIGURE 4: Homography relations between gamma and vision images.

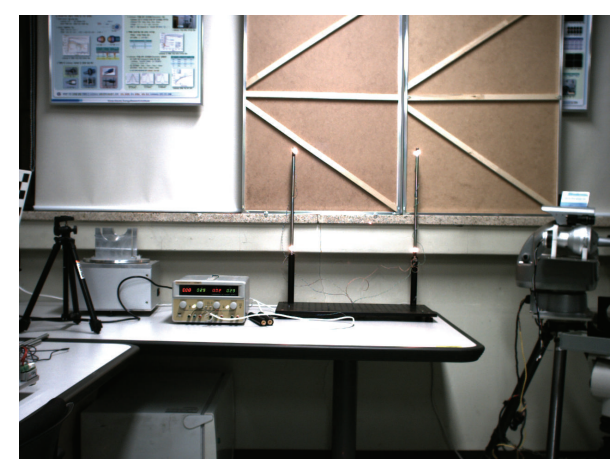

(a)

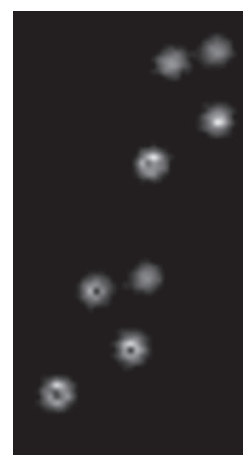

(b)

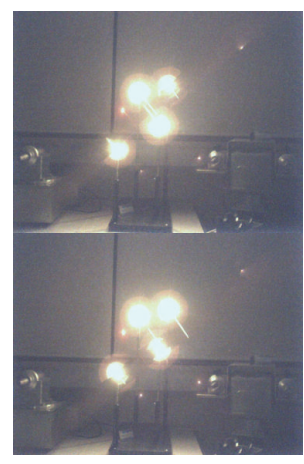

(c)

FIGURE 5: Gamma and vision images of four LED lights: (a) calibration setup, (b) gamma image (top: $J_{\mathrm{L}}$; bottom: $J_{\mathrm{R}}$ ), and (c) vision image (top: $I_{\mathrm{L}}$; bottom: $I_{\mathrm{R}}$ ).

vision images $\left(J_{\mathrm{L}}\right.$ and $\left.I_{\mathrm{L}}\right)$ and the other is between right gamma and vision images $\left(J_{\mathrm{R}}\right.$ and $\left.I_{\mathrm{R}}\right)$.

Let $H_{\mathrm{L}}$ and $H_{\mathrm{R}}$ be the left and the right homography of the gamma and vision cameras, respectively. As shown in Figure $4, H_{\mathrm{L}}$ is the homography from $I_{\mathrm{L}}$ to $J_{\mathrm{L}}$, and $H_{\mathrm{R}}$ is the homography from $I_{R}$ to $J_{R}$. To transform the vision images to gamma images, $I_{\mathrm{L}}$ and $I_{\mathrm{R}}$ are multiplied by $H_{\mathrm{L}}$ and $H_{\mathrm{R}}$, respectively, as follows, and then the results are homogeneously equal to the gamma image:

$$
\begin{aligned}
& J_{\mathrm{L}} \cong H_{\mathrm{L}} I_{\mathrm{L}}, \\
& J_{\mathrm{R}} \cong H_{\mathrm{R}} I_{\mathrm{R}} .
\end{aligned}
$$

Because two sensor's images captured from the same capture position, for example, from $I_{\mathrm{L}}$ and $J_{\mathrm{L}}$, have the same coordinate origin, $2 \mathrm{D}$ homography relation is enough to transform one image plane to the other.

To calculate the planar homography matrix $H$, we need at least four corresponding image points between the gamma and vision images. This means that we need to know at least four sets of 2D image coordinates of any object which can be captured in both gamma and vision cameras. Fortunately, the gamma detection sensor is designed to respond to visible light. Thus we can use four-point light sources to find the homography.

Figure 5 shows an example of capturing four corresponding image points between the gamma and vision cameras. Four LED light sources are fixed in front of the cameras as calibration objects and gamma and vision images of the light sources are obtained. Both in gamma and vision images, the image intensity of the LED sources is higher than those of their neighboring pixels. We apply the Gaussian fitting method [10] to get the exact coordinates of the corresponding points and calculate the homography $H_{\mathrm{L}}$ and the homography $H_{\mathrm{R}}$ using a linear algebra equation.

$H_{\mathrm{L}}$ and $H_{\mathrm{R}}$ are $3 \times 3$ matrices, but their scale is unknown. Therefore, using four pairs of matching points between gamma and vision images, we can compute eight unknown 

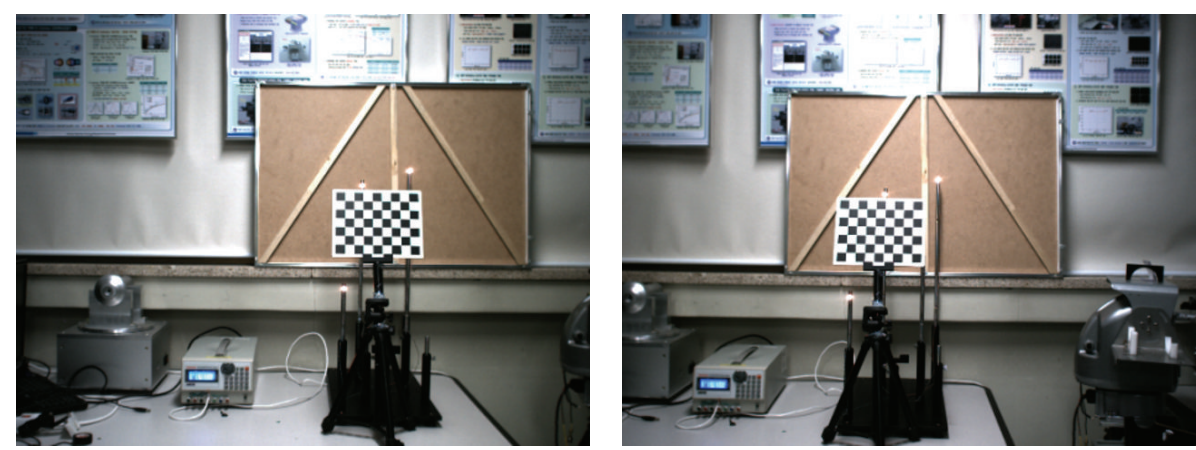

(a)
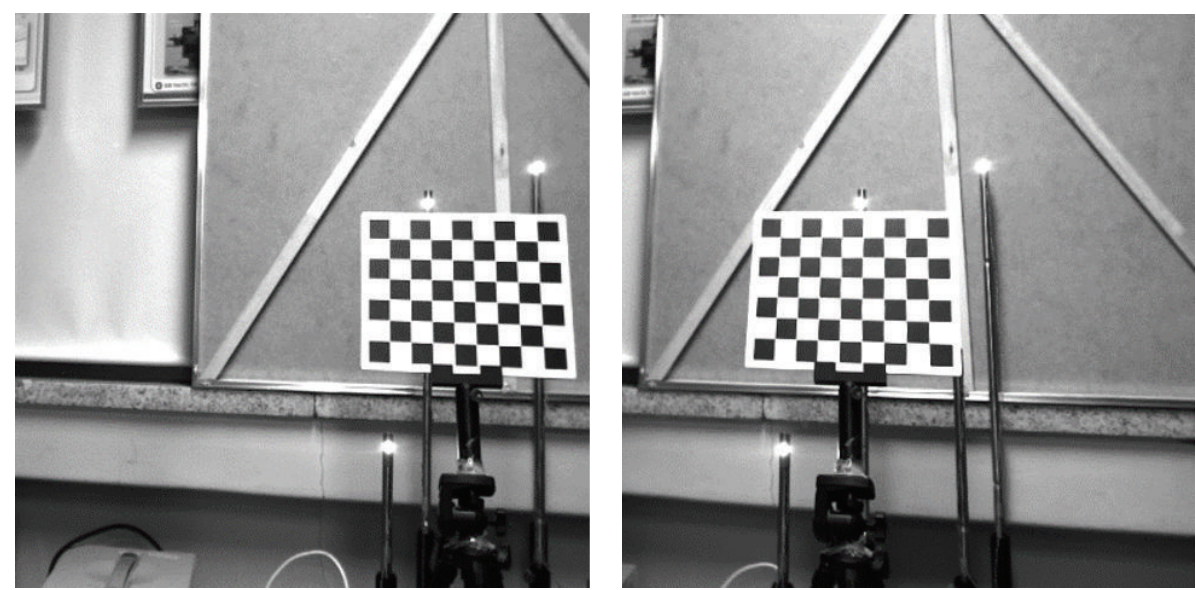

(b)

FIGURE 6: Example of generating calibration pattern images from the view of the stereo gamma camera: (a) left and right vision images and (b) left and right gamma images.

parameters of the homography. Equation (2) shows homography $H_{\mathrm{L}}$ and homography $H_{\mathrm{R}}$ computed by the images in Figure 5:

$$
\begin{aligned}
& H_{\mathrm{L}}=\left[\begin{array}{ccc}
0.53197 & -0.02558 & -164.897 \\
-0.02713 & 0.57747 & -9.0443 \\
-1.7225 \times 10^{-4} & -1.1717 \times 10^{-4} & 1.0
\end{array}\right] \\
& H_{\mathrm{R}}=\left[\begin{array}{ccc}
0.52141 & -0.023501 & -165.795 \\
-0.04064 & 0.52391 & -8.2434 \\
-2.0699 \times 10^{-4} & -2.3451 \times 10^{-4} & 1.0
\end{array}\right] .
\end{aligned}
$$

Figure 6 shows an example of applying the $H_{\mathrm{L}}$ and $H_{\mathrm{R}}$ to the vision images. In Figure 6(a), left and right vision images $I_{\mathrm{L}}$ and $I_{\mathrm{R}}$ are captured in $640 \times 480$ resolution. Using two homography matrices, virtual gamma images $J_{\mathrm{L}}$ and $J_{\mathrm{R}}$ are obtained in $400 \times 400$ resolution. Each pair of left and right images shares the same coordinate origin; thus, there are only scale and rotation transformation between the vision and gamma images (ideally speaking, there is no rotation transformation if the coordinate axes of the two sensors are parallel).

In Figure 7, more light sources are used to calibrate the homography between the two stereo cameras. Images of eight
LED light points are captured in the images from both the vision and gamma cameras. Images in Figure 7(a) show the original captured images of light points from the vision and gamma cameras. Homography relation between the left and right sensors is shown in (3). In Figure 7(c) are virtual gamma images obtained from the homography relation:

$$
\begin{aligned}
& H_{\mathrm{L}}=\left[\begin{array}{ccc}
0.951368 & 0.012253 & -387.186 \\
0.043855 & 0.905885 & -230.053 \\
3.1221 \times 10^{-4} & 4.2492 \times 10^{-5} & 1.0
\end{array}\right] \\
& H_{\mathrm{R}}=\left[\begin{array}{ccc}
0.826863 & 0.025824 & -307.124 \\
0.017759 & 0.821441 & -163.538 \\
1.9185 \times 10^{-4} & 2.0055 \times 10^{-5} & 1.0
\end{array}\right] .
\end{aligned}
$$

\section{Stereo Calibration of Vision and Gamma Cameras}

In the field of computer vision, calibration of a stereo vision camera is a general but important task. Many calibration algorithms have been introduced; however, currently Zhang's method is widely used. To use Zhang's calibration method, it is recommended to capture at least six images of a calibration pattern which usually consists of checkerboard patterns. 

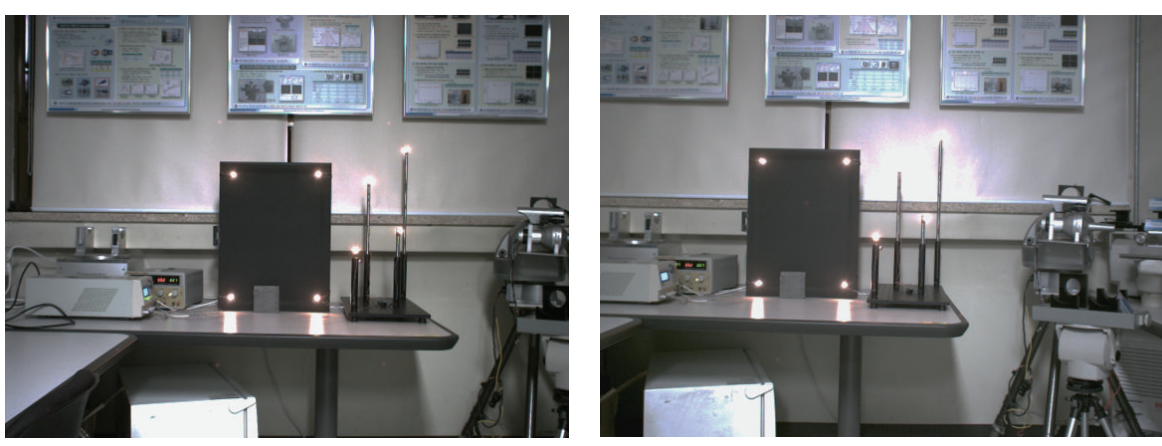

(a)
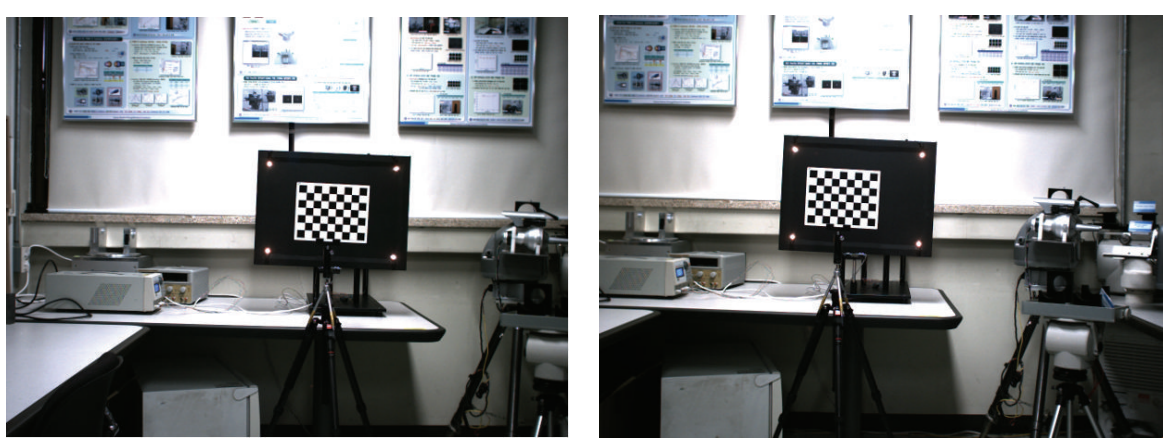

(b)
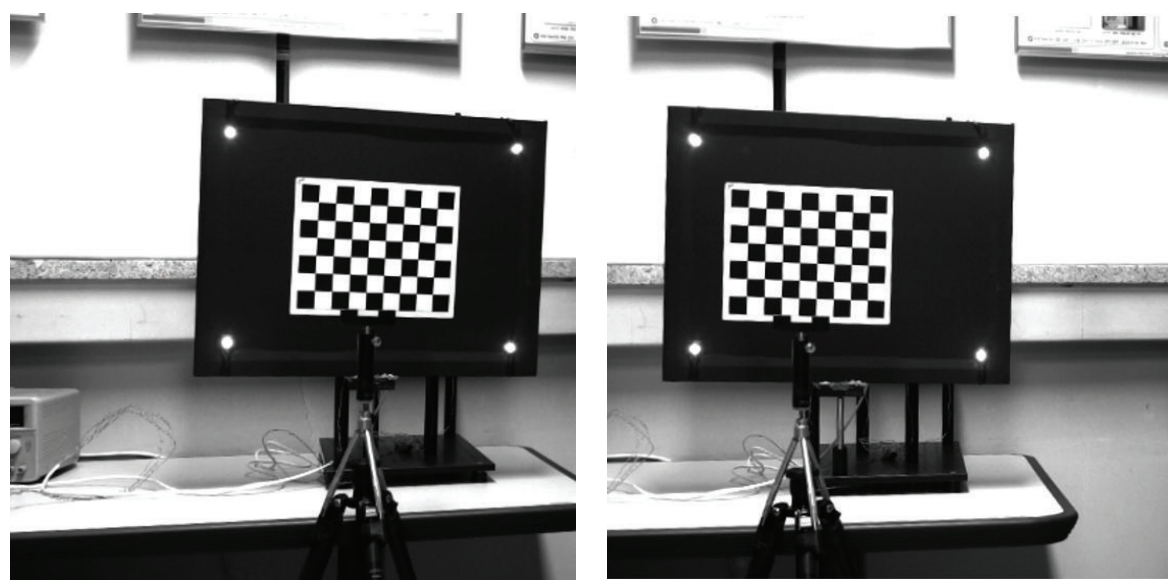

(c)

FIGURE 7: Example of generating calibration pattern images from the view of the stereo gamma camera: (a) left and right vision images and (b) left and right gamma images.

Zhang's calibration algorithm is available in several computer vision libraries such as OpenCV and MATLAB. In this paper, we also use the OpenCV computer vision library for calibrating the vision and gamma cameras.

Obtaining the images of the calibration pattern from the gamma camera is a main problem of this paper. It is because that the gamma camera cannot capture the images of the printed calibration pattern. To solve this problem we use the homography relationship between the gamma and vision cameras. Once several images $I_{\mathrm{L}}$ and $I_{\mathrm{R}}$ of the calibration board from the vision camera are obtained, these images are transformed by $H_{\mathrm{L}}$ and $H_{\mathrm{R}}$ to generate virtual calibration images $J_{\mathrm{L}}$ and $J_{\mathrm{R}}$ from the view of the left and right gamma cameras. Then the transformed images are used as input of Zhang's algorithm to obtain the stereo calibration parameters of the stereo gamma camera. The whole stereo calibration process is shown in Figure 8. The intrinsic and extrinsic parameters of both left and right radiation detectors are depicted in Tables 2 and 3. Here, $R_{x}, R_{y}$, and $R_{z}$ represent the rotation matrix, and $t_{x}, t_{y}$, and $t_{z}$ represent the translation components.

\section{Distance Measurement to Radioactive Sources}

In order to evaluate the accuracy of the proposed calibration method, we performed distance measurement experiments using the calibrated stereo gamma camera. The main 
TABLE 2: Intrinsic parameters of the left and right gamma cameras.

\begin{tabular}{lcccc}
\hline Camera & Focal length $\left(f_{x}\right)$ & Focal length $\left(f_{y}\right)$ & Principal point $\left(C_{x}\right)$ & Principal point $\left(C_{y}\right)$ \\
\hline Left camera & 1048.01 & 1044.52 & 302.31 & 297.64 \\
Right camera & 1048.92 & 1044.95 & 310.61 & 302.64 \\
\hline
\end{tabular}

Step 1. Capture LED light and find homography

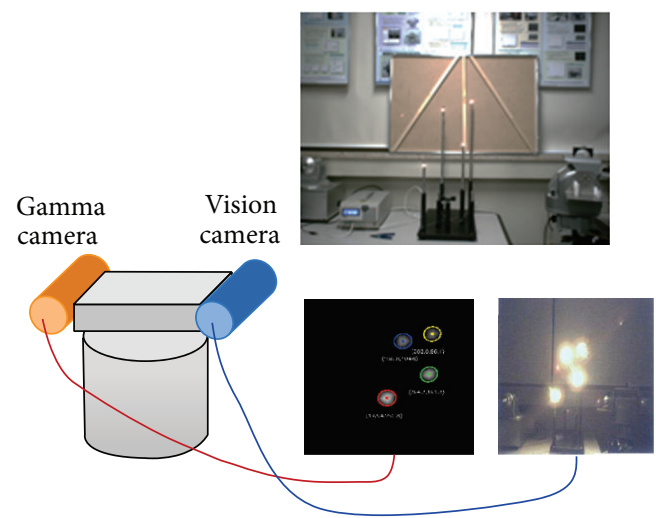

Step 2. Obtain several visual images of a calibration pattern

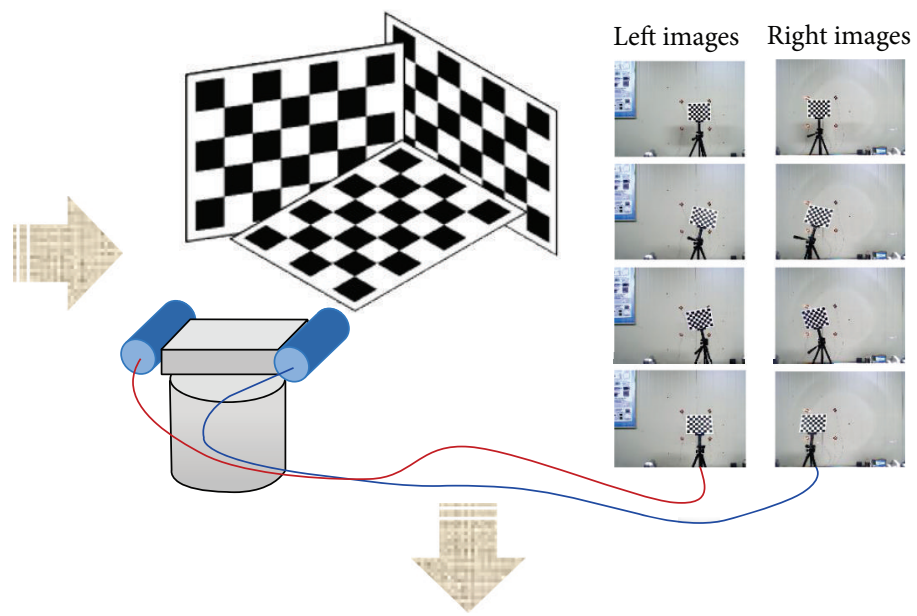

Step 4. Apply Zhang's method to virtual gamma images

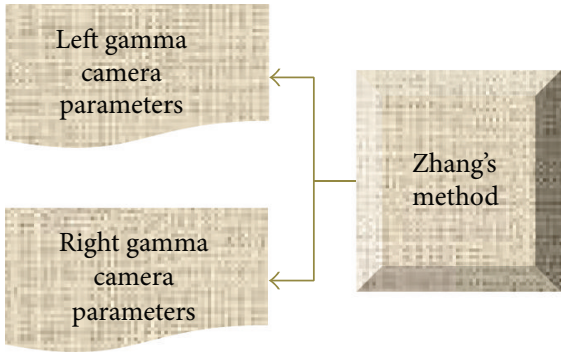

Step 3. Apply $H_{\mathrm{L}}$ and $H_{\mathrm{R}}$ to the left and right vision images
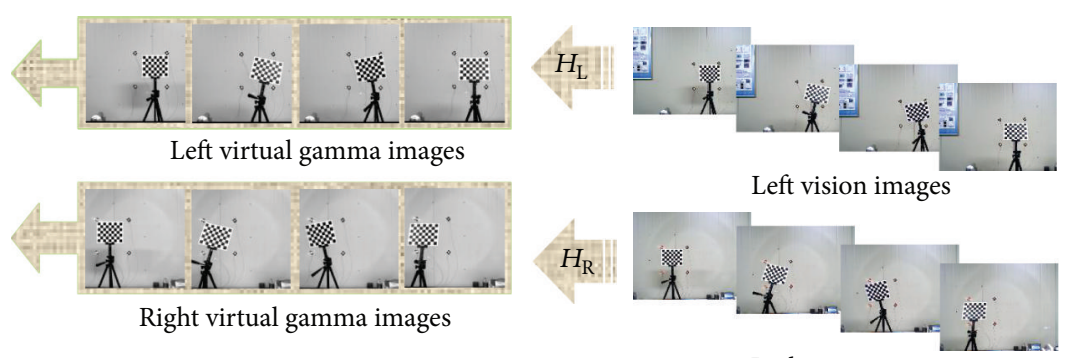

Right vision images

FIGURE 8: The complete processes of stereo gamma camera calibration.

TABle 3: Extrinsic parameters of the right gamma camera with respect to the left camera.

\begin{tabular}{lcccc}
\hline$R_{x}$ & $R_{y}$ & $R_{z}$ & $t_{x}$ & $t_{y}$ \\
\hline 0.00783 & -0.04690 & 0.00209 & -51.03 & -1.14 \\
\hline
\end{tabular}

approach of this is to calculate distances to the previously used bright LED spots which are installed at already known distance. Two kinds of experimental setup "P4" and "P8" are used in distance measurement which are shown already in Figures 5 and 7. In the first experimental setup, four LEDs are placed on different positions as shown in Figure 5(a). In the second experimental setup, eight LEDs are placed on different positions as shown in Figure 7(a).

The positions of the light spot are measured using a Bosch GLM 250 VF Professional laser rangefinder. After gamma camera calibration, the intrinsic and extrinsic camera parameters can be obtained and they are used to calculate left and right perspective projection matrices (PPM). Figure 9 shows left and right stereo gamma images where image processing methods such as adaptive thresholding, contour detection, and ellipse fitting are applied to find the center points $((u, v)$ and $(i, j))$ of each LED light spot of the "P4" setup. Essential and fundamental matrices can also be calculated using the camera parameters according to (4) and the epipolar geometry is applied to identify the corresponding matching points of left and right gamma images. These correspondences are represented with identical colors in Figure 9:

$$
\begin{aligned}
& E=R[t]_{x} \\
& F=K^{\prime T^{-1}} E K^{-1} .
\end{aligned}
$$

$E$ and $F$ represent the essential and fundamental matrices of the stereo gamma camera, respectively. $R[t]_{x}$ is the cross product of the extrinsic camera parameters whereas $K^{\prime}$ and $K$ represent the intrinsic parameters of the left and right cameras, respectively. The $3 \mathrm{D}$ coordinates $W(X, Y, Z)$ for 


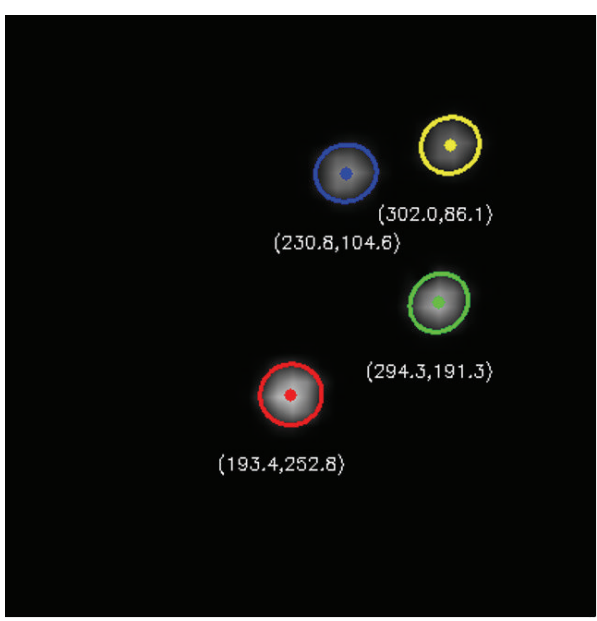

(a)

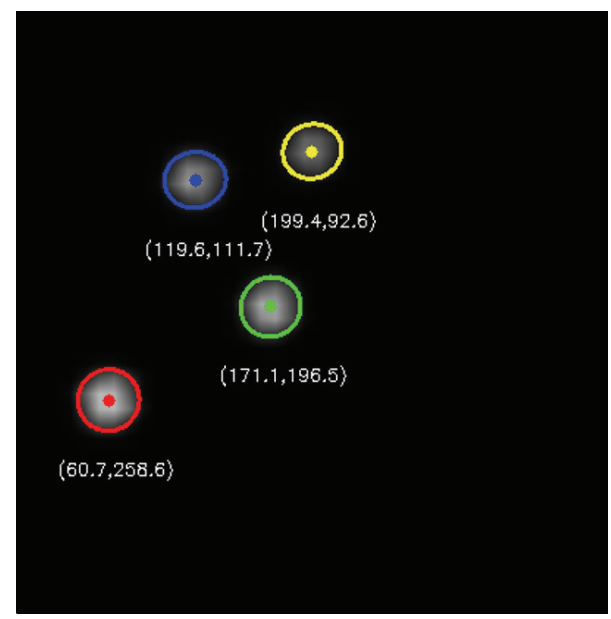

(b)

FIGURE 9: Representing corresponding matching points in left and right gamma images of "P1" setup.

each matching point set can be calculated using a linear equation (5):

$$
A W=Y .
$$

Here $A$ represents a $4 \times 3$ matrix and $Y$ represents a $4 \times 1$ matrix as below. The inverse of matrix $A$ is calculated using the singular value decomposition (SVD) method:

$$
\begin{aligned}
\mathrm{PPM}^{\mathrm{L}} & =\left[\begin{array}{ll}
\left(a_{1}\right)^{T} & a_{14} \\
\left(a_{2}\right)^{T} & a_{24} \\
\left(a_{3}\right)^{T} & a_{34}
\end{array}\right] \\
\mathrm{PPM}^{\mathrm{R}} & =\left[\begin{array}{ll}
\left(b_{1}\right)^{T} & b_{14} \\
\left(b_{2}\right)^{T} & b_{24} \\
\left(b_{3}\right)^{T} & b_{34}
\end{array}\right] \\
A & =\left[\begin{array}{l}
\left(a_{1}-u a_{3}\right)^{T} \\
\left(a_{2}-v a_{3}\right)^{T} \\
\left(b_{1}-i b_{3}\right)^{T} \\
\left(b_{2}-j b_{3}\right)^{T}
\end{array}\right] \\
Y & =\left[\begin{array}{c}
\left(a_{14}-u a_{34}\right) \\
\left(a_{24}-v a_{34}\right) \\
\left(b_{14}-i b_{34}\right) \\
\left(b_{24}-j b_{34}\right)
\end{array}\right] .
\end{aligned}
$$

Figure 10 shows "P4" experimental setup. In this setup, we have done two experiments by changing the position of the four light points as shown in the figure. Figure 10(a) is the same one used in the homography calibration. Figure 10(b) shows four LED lights are placed at the same distance to the gamma camera. Using the two kinds of setup, two
TABLE 4: Distance measurement results of "P4" experimental setup.

\begin{tabular}{lrrrrrc}
\hline \multirow{2}{*}{ Experiments } & \multicolumn{9}{c}{ Test point } & \multirow{2}{*}{ Avg. error (\%) } & \multirow{2}{*}{ Std. Dev. } \\
& 1 & 2 & 3 & 4 & & \\
\hline P4E1 & & & & & & \\
Distance (cm) & 210 & 230 & 250 & 270 & 1.13 & 1.13 \\
Measured (cm) & 207 & 228 & 249 & 274 & & \\
P4E2 & & & & & & \\
Distance (cm) & 300 & 300 & 300 & 300 & 2.41 & 1.59 \\
Measured (cm) & 298 & 305 & 287 & 309 & & \\
\hline
\end{tabular}

distance measurement experiments "P4E1" and "P4E2" are done. As shown in Table 4, the distance measurement is very accurate, where the average percentage error is about $1.13 \%$ in "P4E1" and 2.41\% in "P4E2" experiments. To each point light in Figure 10(a), measurement error is about $1 \sim 4 \mathrm{~cm}$ from camera distance of $210 \sim 270 \mathrm{~cm}$. If the distance to the LED light increases, the measurement error also increases.

We use more LED light points to analyze the accuracy of distance measurement. Figure 11 shows the second experimental setup "P8" using eight LED lights which are fixed in the different distances. As shown in the figure, "point1," "point2," "point6," and "point7" are on the same plane. Four other light points are fixed at the different distances. With this setup, we have done two experiments "P8E1" and "P8E2" by placing the gamma camera at the different distance from this setup. The ground truth distance to each LED light is shown in Table 5.

Figure 12 shows the left and the right gamma images of the "P8E1" experiments. Stereo correspondences between the left and right images are shown in the same colored ellipse models. The center coordinates of the corresponding ellipse models are used to compute the distance of each LED light point. The calibration parameters of these experiments are also derived by using the same setup as described in Section 3 .

Table 5 shows the results of experiments "P8E1" and "P8E2." The average percentile distance error is about $2 \%$ 
TABLE 5: Distance measurement results of "P8" experimental setup.

\begin{tabular}{|c|c|c|c|c|c|c|c|c|c|c|}
\hline \multirow{2}{*}{ Experiments } & \multicolumn{8}{|c|}{ Test point } & \multirow{2}{*}{ Avg. error (\%) } & \multirow{2}{*}{ Std. Dev. } \\
\hline & 1 & 2 & 3 & 4 & 5 & 6 & 7 & 8 & & \\
\hline \multicolumn{11}{|l|}{ P8E1 } \\
\hline Distance $(\mathrm{cm})$ & 320 & 320 & 295 & 310 & 330 & 320 & 320 & 345 & \multirow{2}{*}{2.09} & \multirow{2}{*}{1.63} \\
\hline Measured $(\mathrm{cm})$ & 315 & 314 & 291 & 324 & 328 & 320 & 312 & 360 & & \\
\hline \multicolumn{11}{|l|}{ P8E2 } \\
\hline Distance $(\mathrm{cm})$ & 300 & 300 & 310 & 325 & 300 & 300 & 275 & 290 & \multirow{2}{*}{7.71} & \multirow{2}{*}{5.83} \\
\hline Measured $(\mathrm{cm})$ & 312 & 291 & 261 & 272 & 300 & 324 & 298 & 308 & & \\
\hline
\end{tabular}

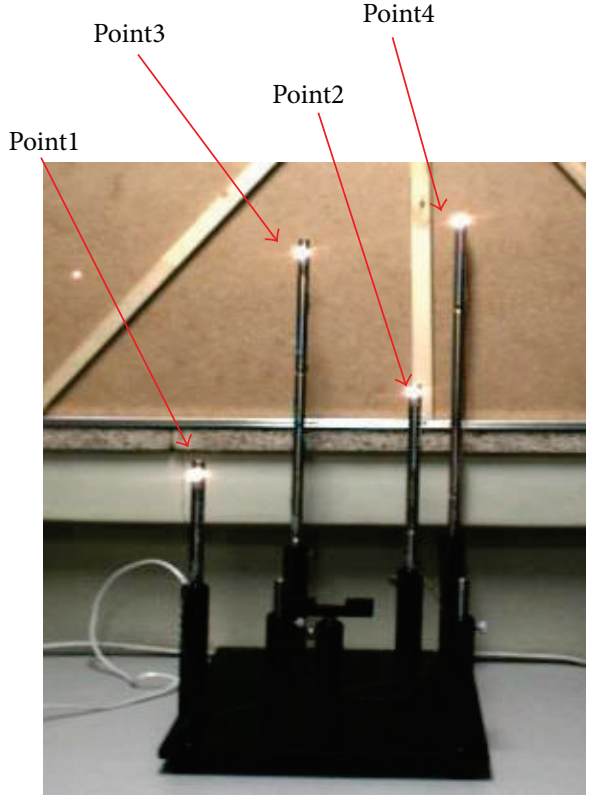

(a)

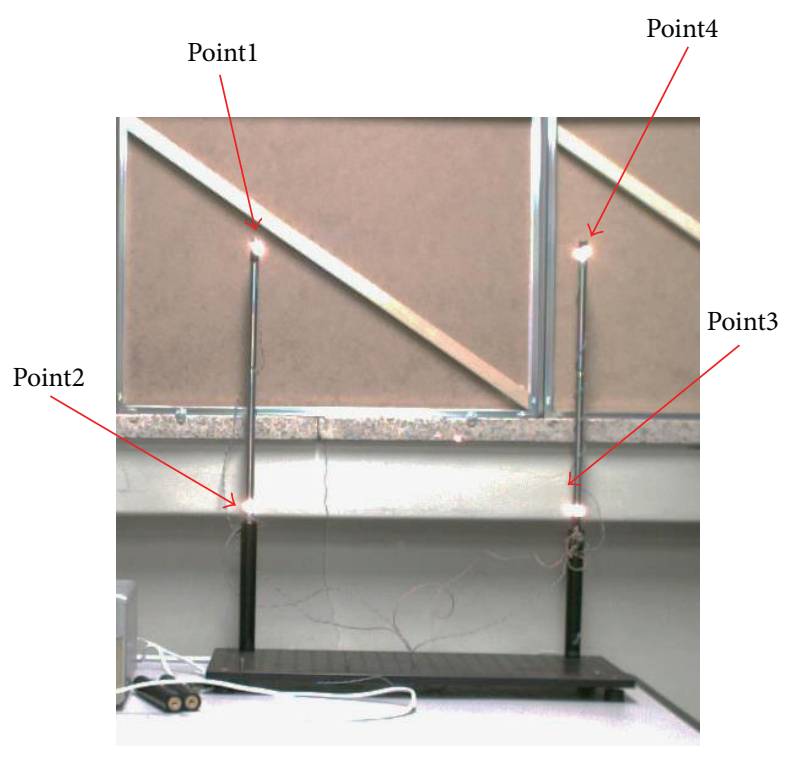

(b)

FIgURE 10: The first experimental setup of the distance measurement using four LED lights: (a) P4E1 and (b) P4E2.

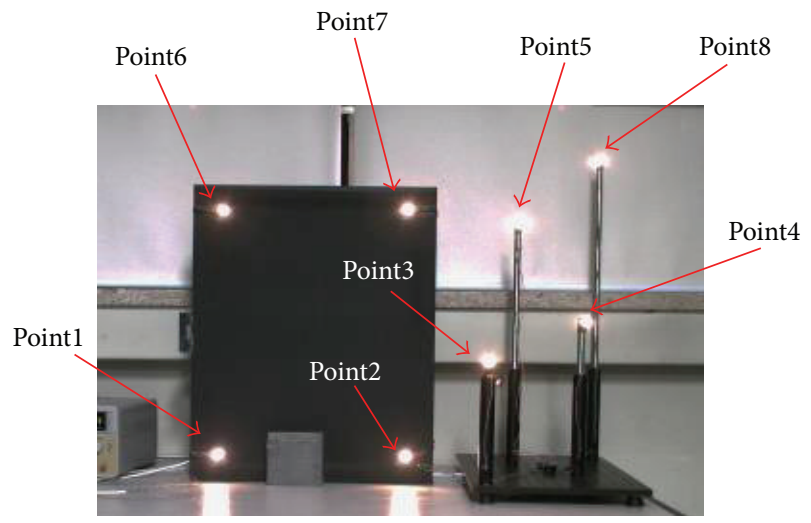

FIGURE 11: Experimental setup of the distance measurement using 8 LED lights. Two experiments "P8E1" and "P8E2" are done using the same setup.

in "P8E1" and 8\% in "P8E2" experiment, respectively. The measurement error is a little higher than that of using four LEDs. This may be caused by using more light points in the computation of the homography relationship. We assume that the centers of the vision and gamma camera are the same, so that light points which are not in the plane can be used for homography computation. However, in reality, the alignment error of the centers of the two cameras can yield higher error not only in the homography computation but also in the distance measurement.

As the final experiment, we use a real radioactive source to obtain stereo gamma images and measure the distance to the source. Figure 13(a) shows a facility for general purpose experiments using a single radioactive source. In this facility, a radioactive source of Cs-137 exposes gamma radiation of $100 \mathrm{mSv} / \mathrm{h}$ at $2 \mathrm{~m}$ distance. As shown in the figure, the gamma source is exposed as a point source. We change the position of the stereo gamma camera module from $150 \mathrm{~cm}$ to $400 \mathrm{~cm}$ and measure the distance to the source using stereo gamma images captured in each distance.

Figure 13(b) shows gamma images captured from the stereo gamma camera. The position difference in stereo images becomes smaller when the distance to the radioactive source becomes farther due to the parallel stereo geometry. In each distance, the center coordinates of the gamma source are 
TABLE 6: Distance measurement results to real gamma.

\begin{tabular}{lcccccccc}
\hline Distance $(\mathrm{cm})$ & 150 & 200 & 250 & 300 & 350 & 400 & Avg. error $(\%)$ & Std. Dev. \\
\hline Measured $(\mathrm{cm})$ & 149.9 & 194.1 & 249.8 & 300.4 & 352.2 & 410.9 & 3.29 & 3.98 \\
\hline
\end{tabular}

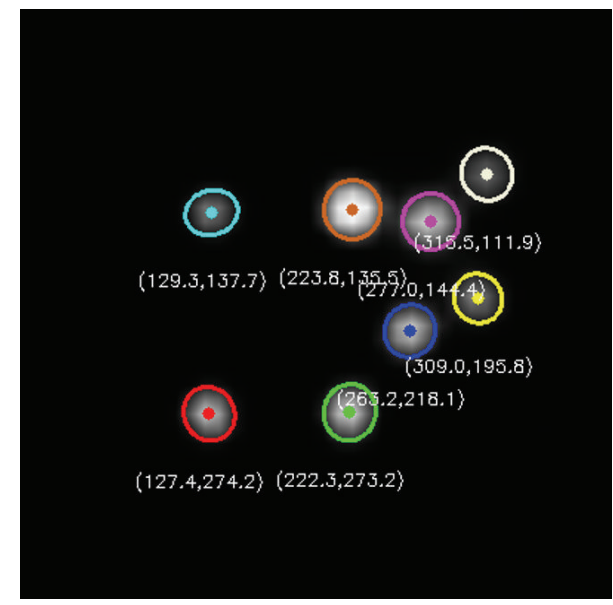

(a)

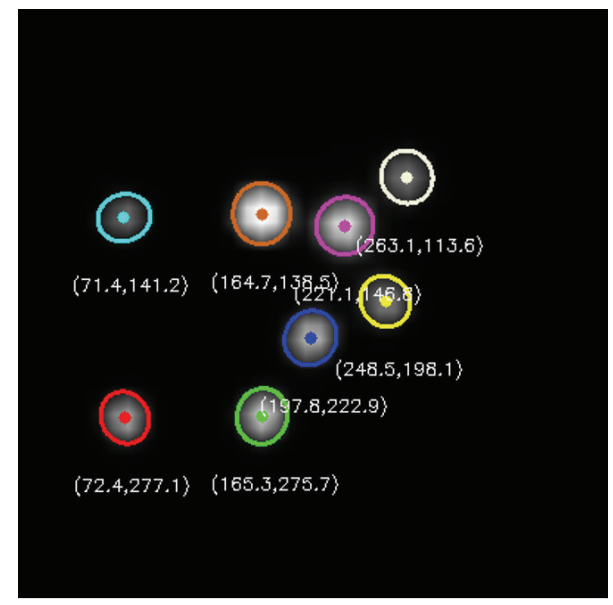

(b)

FIGURE 12: Corresponding points in left and right gamma images of 8 LED points.

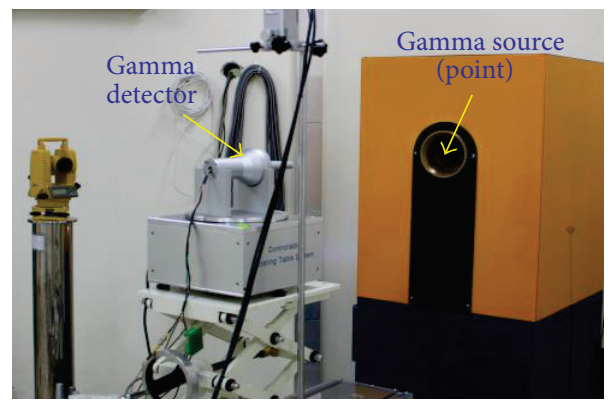

(a)

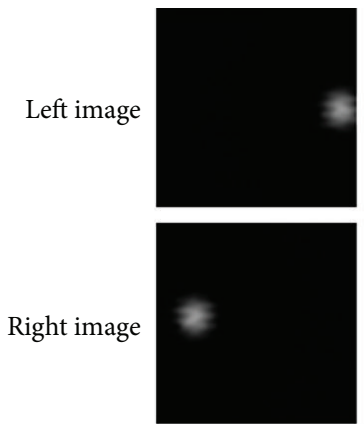

$150 \mathrm{~cm}$
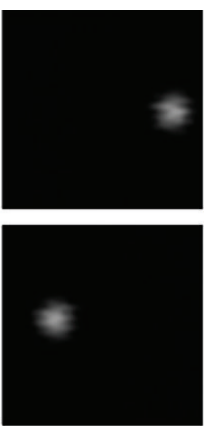

$200 \mathrm{~cm}$
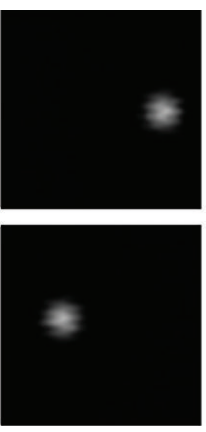

$250 \mathrm{~cm}$
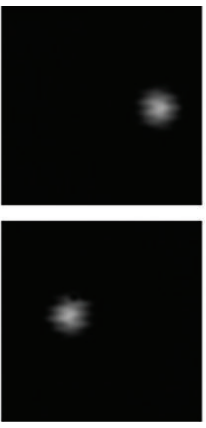

$300 \mathrm{~cm}$
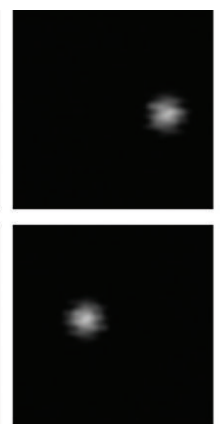

$350 \mathrm{~cm}$
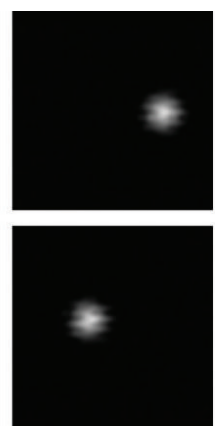

$400 \mathrm{~cm}$

(b)

FIGURE 13: Stereo gamma image acquisition using a real radiation source: (a) experimental facility and (b) stereo gamma image at different measurement distances. 
extracted and the real distances to the source are calculated as in Table 6. Average percentage error is about $3.29 \%$ and it is reasonably accurate results.

\section{Conclusion}

In this paper, we proposed a new method to calibrate stereo radiation detection devices using planar homography relationship. Because it is impossible to capture the calibration pattern images from a gamma camera, we use the planar homography relationship between gamma vision cameras to generate virtual calibration images. Bright LED point lights are used as calibration objects and a gamma camera is used as a radiation detection device. A set of calibration pattern images captured from a vision camera are converted into gamma images by applying homography relationships. Zhang's calibration method is applied to calibrate the gamma camera afterwards. We performed distance measurement experiments using the calibrated gamma camera to check the accuracy of the proposed method. The $3 \mathrm{D}$ coordinates of LED point lights and a real gamma radiation source are obtained with high accuracy using stereo gamma images.

\section{Conflict of Interests}

The authors declare that there is no conflict of interests regarding the publication of this paper.

\section{Acknowledgments}

This work was supported by the Civil Military Technology Cooperation Center and the Korea Atomic Energy Research Institute.

\section{References}

[1] W. Lee and D. Wehe, "3D position of radiation sources using an automated gamma camera and ML algorithm with energydependent response functions," Nuclear Instruments and Methods in Physics Research A, vol. 531, no. 1-2, pp. 270-275, 2004.

[2] T. E. Peterson and L. R. Furenlid, "SPECT detectors: the Anger Camera and beyond," Physics in Medicine and Biology, vol. 56, no. 17, pp. R145-R182, 2011.

[3] H. Kwon, J. Park, and A. C. Kak, "A new approach for active stereo camera calibration," in Proceedings of the IEEE International Conference on Robotics and Automation (ICRA '07), pp. 3180-3185, April 2007.

[4] H. Yu and Y. Wang, "An improved self-calibration method for active stereo camera," in Proceedings of the 6th World Congress on Intelligent Control and Automation (WCICA '06), vol. 1, pp. 5186-5190, June 2006.

[5] Z. Zhang, "A flexible new technique for camera calibration," IEEE Transactions on Pattern Analysis and Machine Intelligence, vol. 22, no. 11, pp. 1330-1334, 2000.

[6] S.-Y. Park and G. G. Park, "Active calibration of cameraprojector systems based on planar homography," in Proceedings of the 20th International Conference on Pattern Recognition (ICPR '10), pp. 320-323, August 2010.

[7] G.-Q. Wei and S. D. Ma, "Complete two-plane camera calibration method and experimental comparisons," in Proceedings of the IEEE 4th International Conference on Computer Vision, pp. 439-445, May 1993.

[8] Y. G. Hwang, N. H. Lee, J. Y. Kim, and S. H. Jeong, “The study for the performance analysis of the stereo radiation detector," in Proceedings of the International Conference on Electronics, Information and Communication (ICEIC '15), 2015.

[9] P. T. Durrant, M. Dallimore, I. D. Jupp, and D. Ramsden, "The application of pinhole and coded aperture imaging in the nuclear environment," Nuclear Instruments and Methods in Physics Research A, vol. 422, no. 1-3, pp. 667-671, 1999.

[10] R. Hartley and A. Zisserman, Multiple View Geometry in Computer Vision, Cambridge University Press, Cambridge, UK, 2004. 


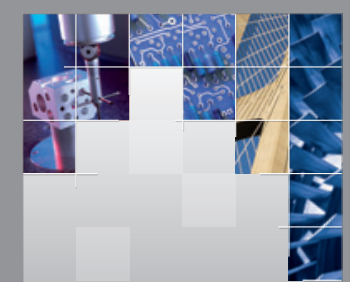

\section{Enfincering}
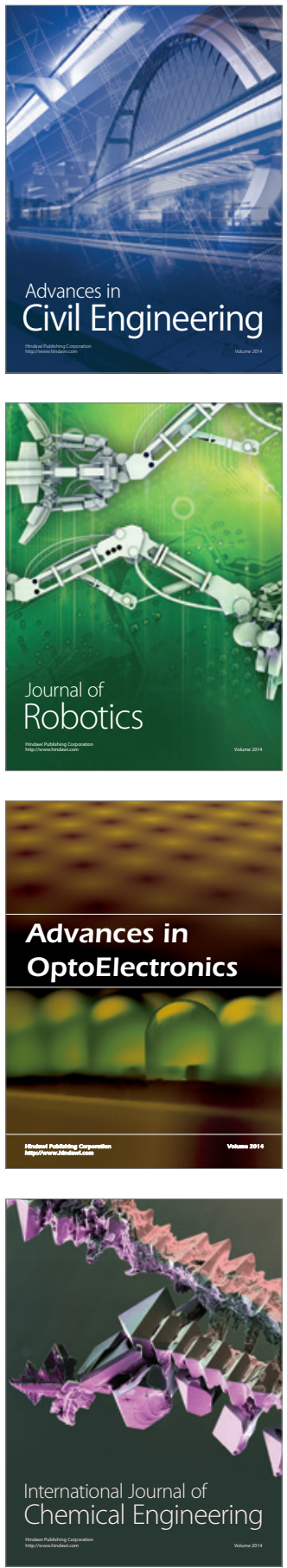

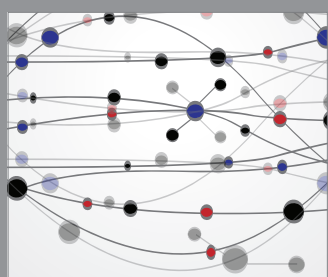

The Scientific World Journal

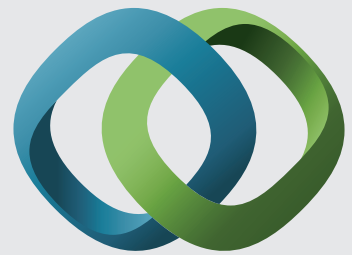

\section{Hindawi}

Submit your manuscripts at

http://www.hindawi.com
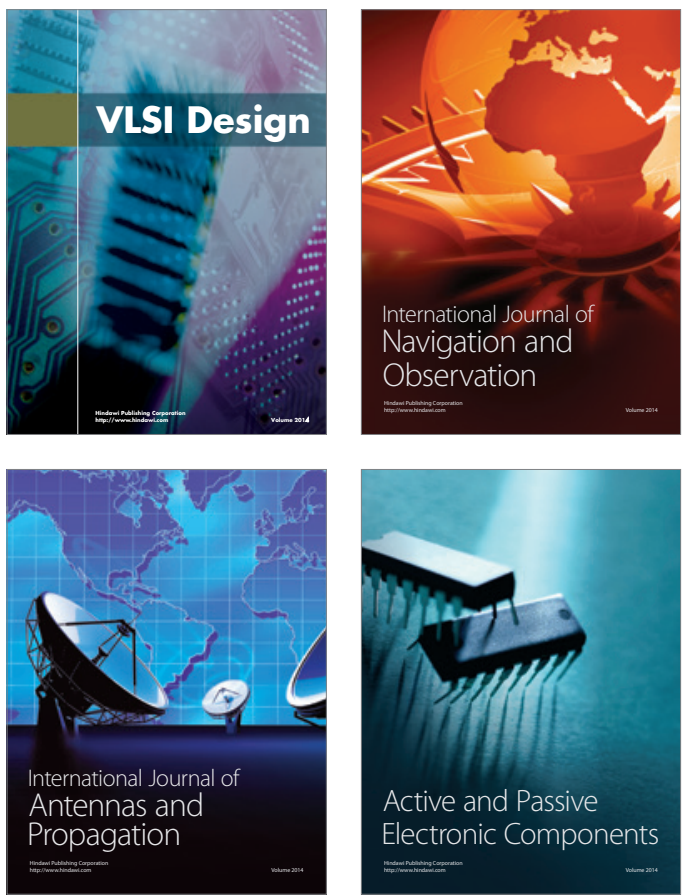
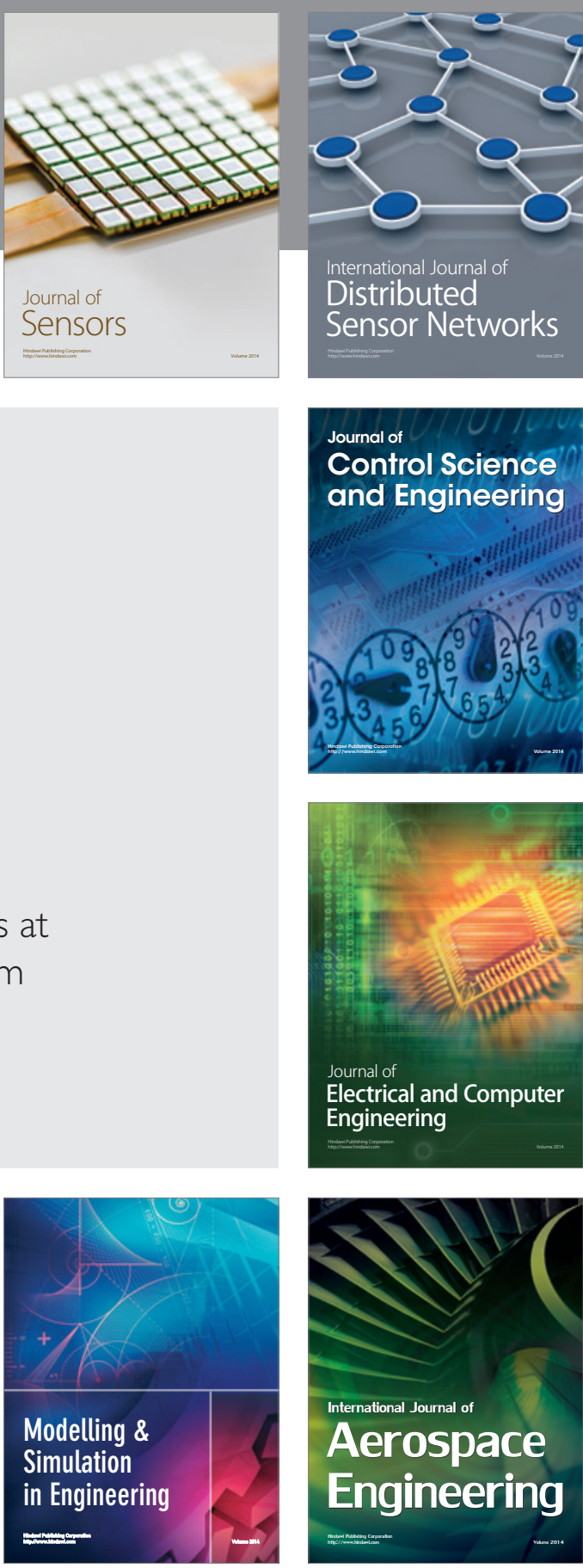

International Journal of

Distributed

Sensor Networks

Journal of

Control Science

and Engineering
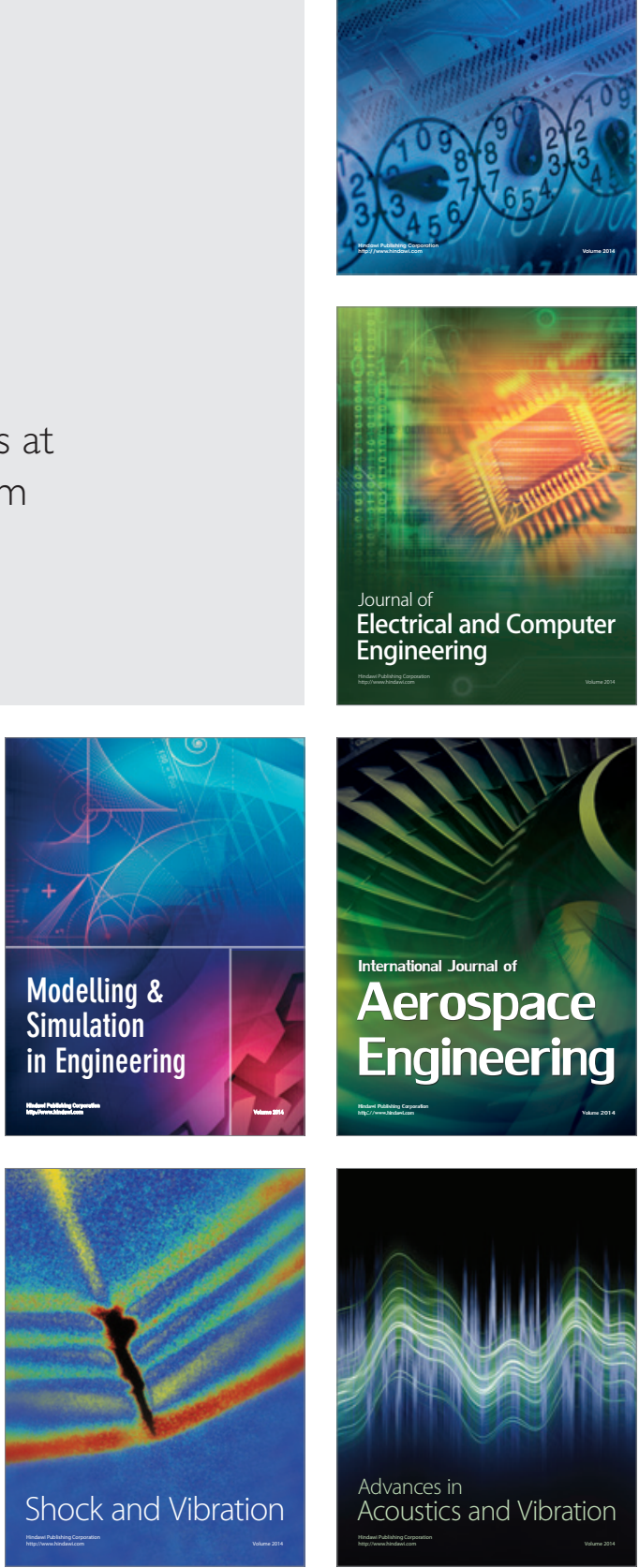